\title{
Algorithm for Reconstructing a 3D Model of the Aortic Root Using Uniform Crushing of CT Images
}

\author{
DOI: $10.17691 / \mathrm{stm} 2018.10 .4 .01$
}

Received March 15, 2018

\author{
K.U. Klyshnikov, Researcher, Laboratory of New Biomaterials, Experimental \\ and Clinical Cardiology Department; \\ E.A. Ovcharenko, PhD, Head of the Laboratory of New Biomaterials, Experimental \\ and Clinical Cardiology Department; \\ V.I. Ganyukov, MD, DSc, Professor, Head of the Laboratory of Interventional Diagnostics \\ and Treatment of Atherosclerosis;
}

R.S. Tarasov, MD, DSc, Head of the Laboratory of Reconstructive Surgery of Multifocal Atherosclerosis;

A.N. Kokov, MD, PhD, Head of the Laboratory of Radionuclide and Tomographic Diagnostics;

L.S. Barbarash, MD, DSc, Professor, Academician of the Russian Academy of Sciences, Chief Researcher

Research Institute for Complex Issues of Cardiovascular Diseases, 6 Sosnovy Blvd, Kemerovo, 650002, Russia

The aim of the study was to develop a uniform crushing method to be used in reconstructing a computational grid for 3D models simulating transcatheter aortic valve implantation based on medical images (DICOM) and mathematical processing and then to compare this novel approach with the currently used polygon and CAD methods.

Materials and Methods. The method was developed using the clinical and imaging data of patient N., 68 years old, who underwent transcatheter aortic valve replacement. The images were taken from multispiral computed tomography. The aortic root models were reconstructed using three methods: the polygons method (Mimics, Belgium), the CAD method (NX 9.0, Germany) and the author's algorithm implemented in MATLAB (USA). Quality assessment of the reconstructed models was performed by numerical simulation using the Abaqus/ CAE 6.14 (USA) engineering analysis reproducing two pressure load scenarios: the systole and the diastole.

Results. Evaluation of the reconstructed models of the aortic root showed that the proposed numerical method allows one to segment the object (aortic root) into elements, which are more homogeneous as compared to the polygon method or the CAD method. Thus, in the polygon method, 128,452 tetrahedrons (pyramids, C3D4) were used; in the CAD method, 28,456 hexahedrons (parallelograms, C3D8) were used; and in the case of the numerical method - 24,644 identical C3D8 elements were used. The results of the numerical simulations also differed: in the polygon method, the von Mises maximum was $0.262 \mathrm{MPa}$; for the CAD algorithm, $0.412 \mathrm{MPa}$; and in the numerical method, $0.359 \mathrm{MPa}$. An important criterion of the reconstruction efficiency was the computation time factor: for the polygon method, it was $458.6 \mathrm{~s}$, for the CAD algorithm - $377.2 \mathrm{~s}$ ( $21.5 \%$ less), and for the proposed numerical method $-341.8 \mathrm{~s}$ ( $34.1 \%$ less).

Conclusion. The qualitative and quantitative results of the study demonstrate the usefulness of the proposed algorithm based on hexahedral finite elements in reconstructing a biological object for the purpose of numerical analysis. Using this algorithm in the transcatheter aortic valve implantation modeling process makes it possible to reduce the time of numerical analysis and increase its accuracy, which may improve the quality of preoperative planning.

Key words: finite element method; transcatheter aortic valve implantation; three-dimensional models of biological objects.

\section{Introduction}

One of the most important challenges in cardiac surgery is the preoperative planning of interventions, including the accesses route, the choice of prosthesis, and the prediction of outcomes and complications $[1,2]$.
Planning is of particular importance in minimally invasive operations, where an interventional cardiologist has to assess the nature and extent of the lesion based on the results of instrumental studies - echocardiography (echoCG), multispiral computed tomography (MSCT), or MRI [3-5]. In recent decades, interventions like

Corresponding author: Kirill U. Klyshnikov, e-mail: Klyshnikovk@gmail.com 
percutaneous mitral valve implantation and transcatheter aortic valve implantations (TAVI) have become commonly used procedures worldwide, including Russia [6-8]. Cardiologists plan TAVI interventions based on their own experience, requirements of the manufacturer/regulator, and the protocols recommended by their professional societies. In these arrangements, the real outcome of TAVI can be determined only after the procedure is completed. Therefore, the planning step bears an element of uncertainty, which may negatively impact the treatment outcome and even cause complications like paraprosthetic fistula or conductivity disruption [9], arising from erroneous implant positioning or prosthesis size mismatch. The numerical methods currently used in medicine are considered promising for TAVI planning due to their ability to simulate the implantation procedure in various modifications [10, 11]. There are reports in the literature describing the TAVI numerical analysis, but, in our view, they suffer from an insufficient level of predictive accuracy and a low cost-effectiveness, which prevents their practical use in the clinic $[12,13]$.

Another possible reason for the limited use of numerical methods in TAVI planning can also be the quality of $3 \mathrm{D}$ models of objects involved in the interaction - aortic root, valve prosthesis, native aortic valve, calcifications and degenerative elements. The existing methods of non-destructive structure examination - computer microtomography - allow one to recreate the structure of TAVI prostheses with high accuracy (up to $0.025 \mathrm{~mm}$ ) and to reconstruct them [14]. However, due to high radiation exposure, it is impossible to use these methods in vivo; therefore, for preoperative planning, only "classical" methods of clinical imaging are available - MSCT, MRI, and echoCG.

Reconstruction of 3D computer models for TAVI planning should meet a set of requirements: among them, high accuracy, limited computation time, and medical validity [15]. In the current practice, the process of reconstruction can be completed using two approaches: the most common method of polygons, implemented in commercial software (Amira; Mimics; Slicer 3D), and the CAD method based on computeraided design software (NX; SolidWorks). The choice of the method depends on the accuracy and complexity of the model, but each of them has its own shortcomings, which ultimately influence the simulation results. The method of polygons demonstrates high accuracy, thanks to the large number of elements in the grid nodes, however, this features significantly complicate computer calculations [16, 17]. The CAD method, in contrast, allows you to create a high-quality mesh, but it is less accurate and less cost-effective than the polygon method.

The aim of the study is to develop a uniform crushing method for constructing a computational grid for 3D models of TAVI using mathematical processing of the DICOM data and then to compare the proposed method with the standard polygons and CAD methods.

\section{Material and Methods}

The DICOM data. To develop a method for 3D reconstruction to be used in the numerical analysis of the TAVI procedure, we processed images made in the DICOM format (Digital Imaging Communications in Medicine) of patient N., who was implanted a CoreValve bioprosthesis (Medtronic Inc., USA). According to the protocol, at the preoperative planning stage, the patient underwent MSCT without a contrast material with visualization during systole and diastole using a SOMATOM Sensation 64 tomograph (Siemens, Germany). The scanning direction was craniocaudal with the following characteristics: voltage $-120 \mathrm{kV}$, aperture - $200 \mathrm{~mm}$, pitch factor - $0.7 \mathrm{~mm}$, resolution - $0.625 \mathrm{~mm}$, ECG synchronized, slice thickness $-1 \mathrm{~mm}$.

$3 D$ geometry reconstruction - the polygon method. Based on the DICOM images, the aortic root was reconstructed using the commercial Polygon software adjusted to medical image analysis. To this end, the images were imported into the Mimics medical imaging software (Materialize, Belgium), where the aortic root was reconstructed using the embedded contouring tools. The resulting 3D reconstruction file was then exported in the form of a finite-element mesh into the Abaqus/CAE 6.14 engineering analysis environment (Dassault Systèmes, France).

\section{$3 D$ geometry reconstruction - the CAD method.} The initial DICOM image sets were divided into separate images with the original quality preserved. Among them, groups of "basic" and "guiding" slices were selected (Figure 1 (a), (d)). The "basic" slices were obtained from the axial MSCT scans, i.e. they were directed along the aortic root - the main object of the reconstruction. The image frequency varied depending on the complexity of the specific areas of the object. Thus, the Valsalva's sinuses, the coronary arterial orifices and the fibrous ring of the aortic valve were reconstructed in more detail than the ascending section of the aorta (Figure 1 (b)). The "guiding" slices reflected the sagittal plane and two auxiliary planes passing through the arbitrary longitudinal axis of the aortic root. These slices were used to highlight the internal and external profiles of the object and design its "skeleton". The images thus selected were imported into the NX 9.0 CAD system (Siemens PLM Software, Germany) while maintaining the relative position and scale determined by the resolution of the clinical MSCT (see Figure 1 (b), (e)).

Based on these imported images, a set of drafts were constructed in the planes corresponding to the "basic" and "guiding" slices. The transformation was conducted using CAD tools such as segments, arches, and open splines to form closed contours for the internal and external surfaces of the model ("basic" slices), as well as open "guiding" curves (Figure 1 (c), (f)).

Sequential integration of the drafted elements into thin-walled shells using CAD tools resulted in a set of 


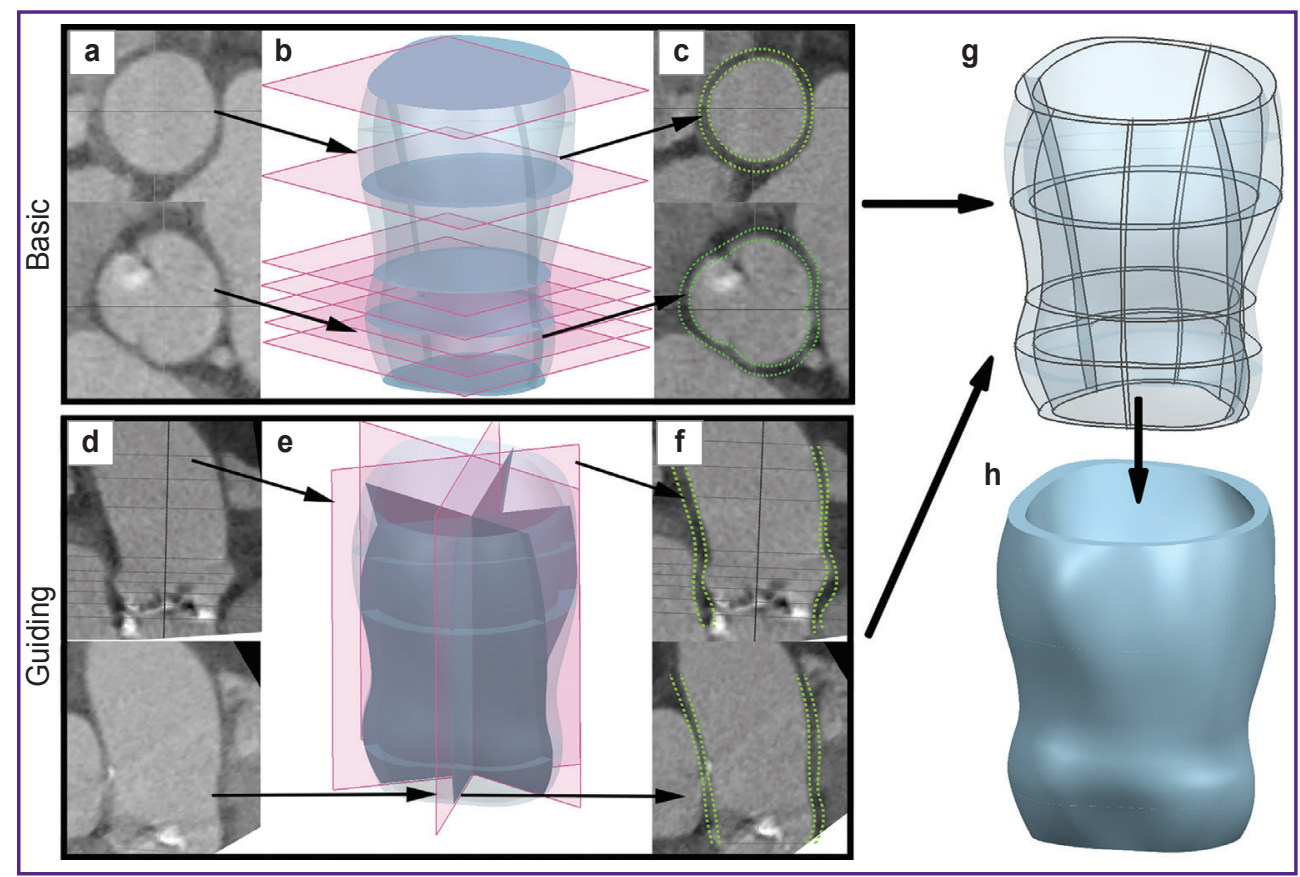

Figure 1. Stages of reconstruction of the aortic root model using the CAD method:

(a) an example of "basic" axial MSCT slices selected for reconstruction; (b) "basic" slices represented by parallel planes in the CAD model; (c) drafting by the "basic" slices; (d) an example of "guiding" MSCT slices; (e) location of the "guiding" slices; (f) drafting by the "guiding" slices; (g) merging the drafts into a single "skeleton"; (h) constructing a 3D solid model by stitching the surface parts derived from the "skeleton"

membranes describing the reconstructed geometry. The subsequent "stitching" of the membranes into a solid produced the sought geometry (Figure $1(\mathrm{~g}),(\mathrm{h})$ ).

$3 D$ geometry reconstruction - the method of uniform crushing. The proposed aortic root reconstruction method is based on the sequential analysis of all available DICOM slices with the algorithmic construction of a hexagonal finite element mesh. The set of images obtained from MSCT was imported into the original script in the MATLAB. The developed algorithm provides for the creation of a 3D matrix of numbers expressed as shades of gray followed by affine transformation aimed to locate the region of interest in the selected coordinate system. Then, binarization is automatically performed according to the Otsu method [18] (Figure 2 (c)). In each binary image, a starting point relating to the inner lumen of the aortic root was generated. From this starting point, the contour was automatically scanned using the Moore algorithm in such a way as to highlight the boundary of the inner lumen of the aortic root, based on different contrasting, after which the center of masses was determined (Figure $2(\mathrm{~d})$ ).

This center of masses is the starting point of radial rays (with a given increment of $1^{\circ}$ ) used to locate intersections with the boundary of the inner contour. The found points of intersection serve as the starting point, where the construction of radial segments reaching the nearest white pixel begins. These segments represent the thickness of the aortic wall, i.e. they constitute the basis for constructing the outer contour of the model. However, it is not always possible to accomplish the segment intersection with a white pixel, because the binarization may leave black areas on some tomograms. In these areas, therefore, the array of segments thicknesses - was supplemented by linear interpolation between two boundary values in the slice and expressed in the cylindrical coordinate system. Thus, based on the obtained contour of the inner lumen of the aortic root and the full array of segments - thicknesses with $1^{\circ}$ increment, the outer contour of the model was reconstructed. In the future, the task may be simplified and reduced to determining the coordinates of the major nodes of the finite elements describing the wall of the aortic root. Thus, the coordinates of the nodes in two adjacent slices are determined; then, they are sequentially combined in a format suitable for import into the Abaqus/CAE 6.14 to result in an unrelated grid (see Figure 2).

Numerical simulation. To test the proposed reconstruction method under the conditions of a numerical experiment, the aortic root wall compliance under normal physiological pressure was simulated: $120 \mathrm{~mm} \mathrm{Hg}$ for systole and $80 \mathrm{~mm} \mathrm{Hg}$ for diastole. The numerical experiment was carried out by the finite element method in the Abaqus/CAE 6.14 software. 


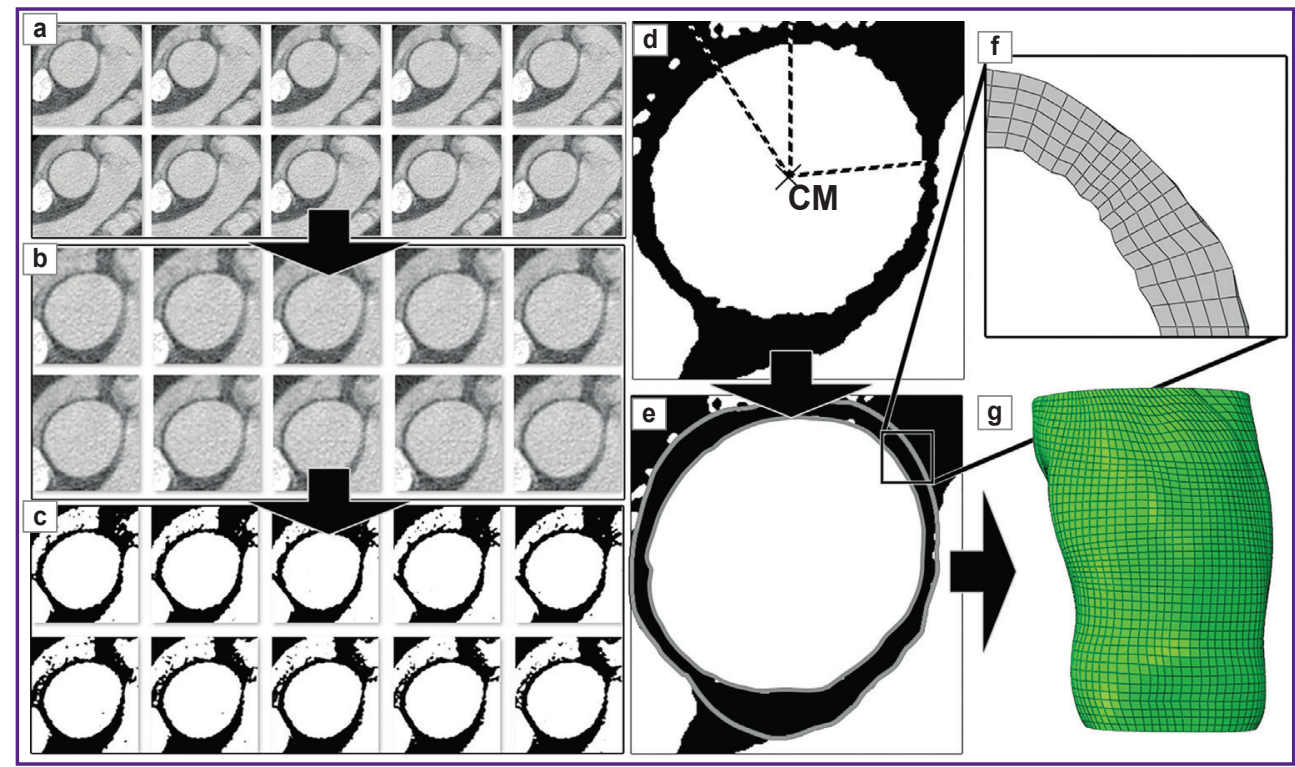

Figure 2. Reconstruction of the aortic root model by the method of uniform crushing: (a) initial tomographic images scanned in the transverse direction; (b) slices transformed by the batch resizing; (c) binarized images; (d), (e) algorithm for searching the inner and outer borders of the aortic root (CM is the center of masses); (f) a finite element mesh in the plane of each slice; (g) sequential merge of finite-element mesh nodes and formation of a 3D unrelated mesh (orphan mesh) in the Abaqus/CAE engineering analysis environment

Figure 3. Resulting finite-element grids and limiting conditions for conducting a numerical experiment in the Abaqus/CAE environment:

(a) grid obtained in the Mimics software; (b) grid created from the CAD-reconstructed model; (c) a finite element mesh obtained by the proposed numerical method; the arrows indicate the load distribution; the cross marks indicate the movement restriction points along the central longitudinal axis (Z); the dark color denotes the areas of the fibrous ring

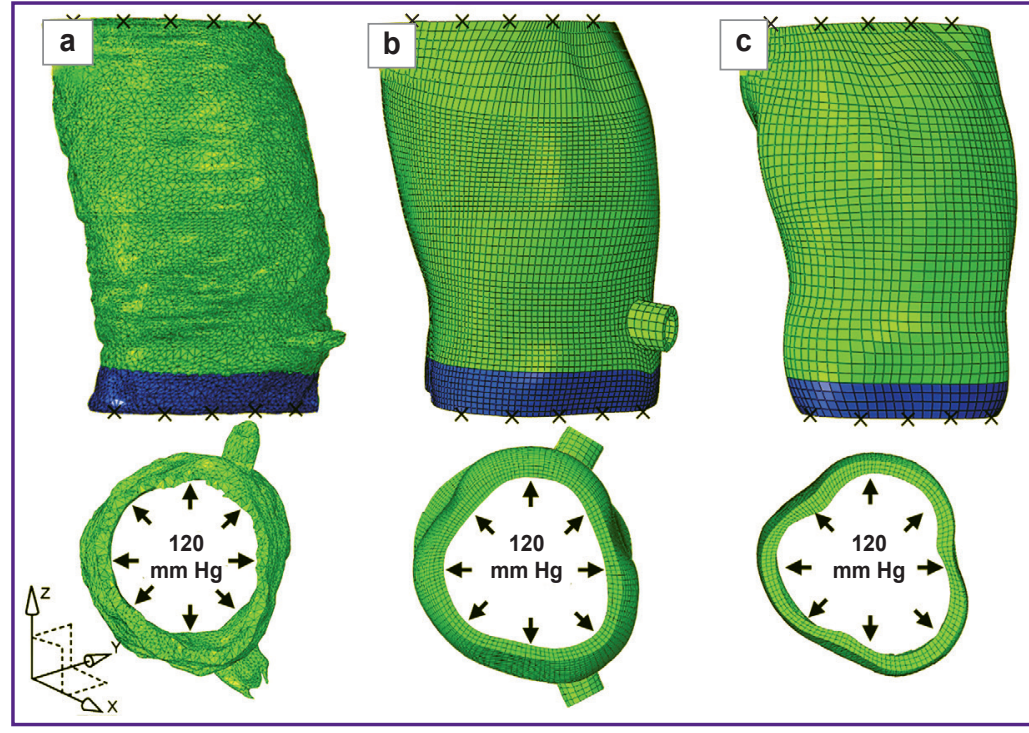

Three aforementioned models of the aortic root were used in the study: a) produced by the polygons method, b) produced by the CAD-method, and c) resulted from the proposed method of uniform crushing (Figure 3 ). A pressure load similar to physiological pressure in the aortic root was applied to the inner surface of the models. The limiting conditions were determined by imposing restrictions on the movement along the central longitudinal axis $(Z)$ in the area of the fibrous ring and sinotubular junction.
The material characteristics of the simulated tissue were obtained from our own data on the physicalmechanical parameters of the native aortic root of cadaveric material [19]. As a result, second-order polynomial models of hyperelastic materials were obtained for the fibrous ring and, separately, for the overlying aortic segments (see Figure 3) with the coefficients shown in the Table.

The load applied to the objects of interest was pseudo-dynamic due to the low rates of pressure 
growth from systole to diastole, therefore the Abaqus/Standard method (not implying explicit time integration) was chosen as a solver. In the course of the modeling, the stress-strain state was assessed by using the von Mises stress score and the index of logarithmic deformation, as well as their qualitative distribution on the respective epures.
Polynomial models of the aortic root tissues used in the Abaqus/CAE environment

\begin{tabular}{lccccccc}
\hline $\begin{array}{c}\text { Areas } \\
\text { of aortic root }\end{array}$ & $\begin{array}{c}\mathrm{C10} \\
(\mathrm{MPa})\end{array}$ & $\begin{array}{c}\mathrm{C01} \\
(\mathrm{MPa})\end{array}$ & $\begin{array}{c}\mathrm{C20} \\
(\mathrm{MPa})\end{array}$ & $\begin{array}{c}\mathrm{C11} \\
(\mathrm{MPa})\end{array}$ & $\begin{array}{c}\mathrm{C02} \\
(\mathrm{MPa})\end{array}$ & $\begin{array}{c}\mathrm{D} 1 \\
(1 / \mathrm{MPa})\end{array}$ & $\begin{array}{c}\mathrm{D2} \\
(1 / \mathrm{MPa})\end{array}$ \\
\hline Fibrous ring & -0.15 & 1.1 & 0.010 & 0.015 & 0.7 & 0.05 & 0.05 \\
\hline Overlying areas & -0.25 & 1.6 & 0.015 & 0.025 & 1.1 & 0.05 & 0.05 \\
\hline
\end{tabular}

Note: C10, C01, C20, C11, C02 - phenomenological coefficients characterizing the shear behavior; D1, D2 - volumetric strain coefficients.

\section{Results and Discussion}

The present results demonstrate that the proposed method of uniform crushing allows one to segment the aortic root model into elements more homogeneous than those in the polygon method and the CAD method. The result is important since insufficient homogeneity can adversely affect the simulation results and even distort them [20]. The obtained data confirm this statement. For example, in the aortic root models based on the proposed algorithm (as well as the CAD method), there were no areas of high von Mises stress gradient, while in the polygonal model such areas were observed. It is worth noting that the occurrence of a high gradient between finite elements can be a consequence of erroneous calculation, since it has no physical explanation - in a homogeneous material, no significant stress gradient can occur between two adjacent points. Thus, in the proposed method (and the CAD method) of reconstruction, there are less calculation errors.

In general, in our numerical experiment, variable quantitative data were obtained. Thus, in the polygons method, the von Mises maximum value was $0.262 \mathrm{MPa}$, and in the CAD algorithm $-0.412 \mathrm{MPa}$. It should be noted that the hexahedral elements used in the CAD calculations and in the proposed method show close overall results, while the mesh obtained in the Mimics software slightly underestimates the stress-strain ratio (Figure 4).

In terms of modeling, an important parameter is the calculation time, i.e. the direct physical time spent by the solver to complete the numerical calculations. This study showed that even in the case of a relatively simple task - modeling the effect of pressure on the aortic root - there are significant differences between the three models used for the reconstruction. Thus, in the polygons method, the calculation time was 458.6, and in the CAD algorithm - 377.2 (21.5\% less), and in the uniform crushing method - 341.8 (34.1\% less). This difference can be due to the different nature of finite elements used in these models, and also to the different number of these elements. Thus, in the polygon method, there were 128,452 tetrahedrons (pyramids C3D4); in the CAD method - 28,456 hexahedrons (parallelograms - C3D8); and in the numerical method, there were 24,644 identical C3D8 elements (see Figure 3 ). The average rib length was $0.99,0.91$, and $0.98 \mathrm{~mm}$, respectively. On the other hand, the use of tetrahedrons is reasonable: tetrahedral grids are capable of reconstructing any geometry, while hexahedral grids require lengthy preparations both in the CAD method and in the numerical algorithm. The differences in the

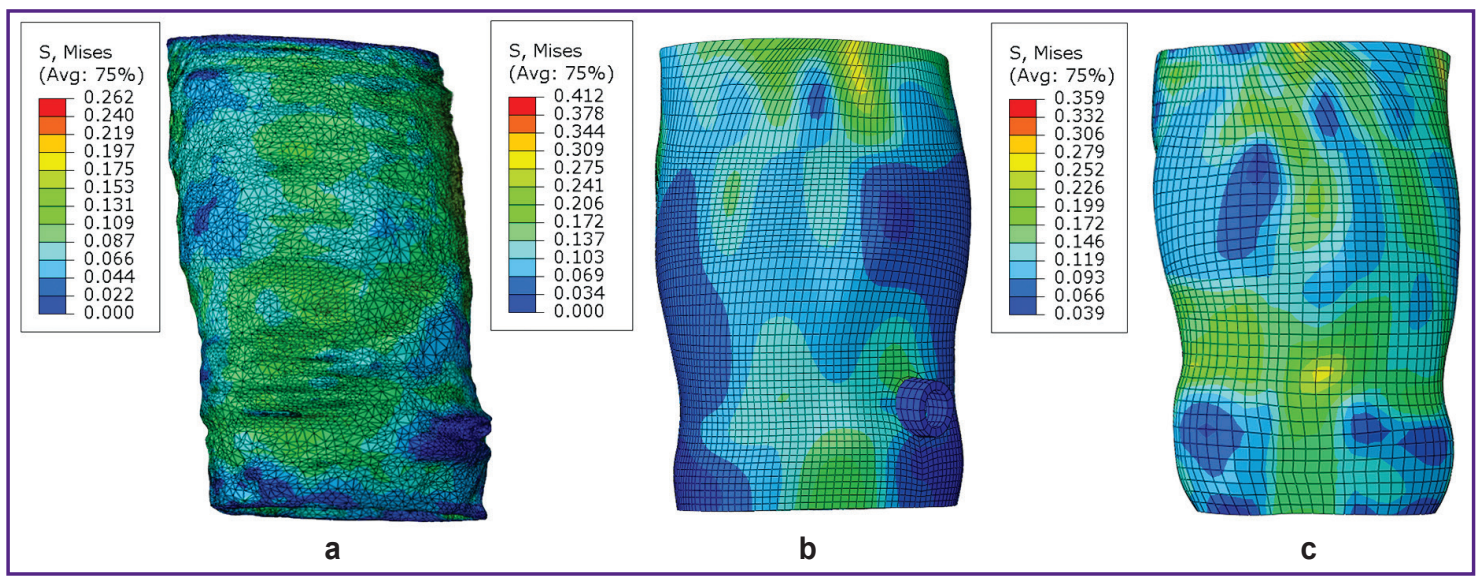

Figure 4. Epure presentation of stress distribution according to von Mises (MPa) in the reconstructed models of the aortic root:

(a) by the method of polygons; (b) by the CAD method; (c) by using the proposed method of uniform crushing 


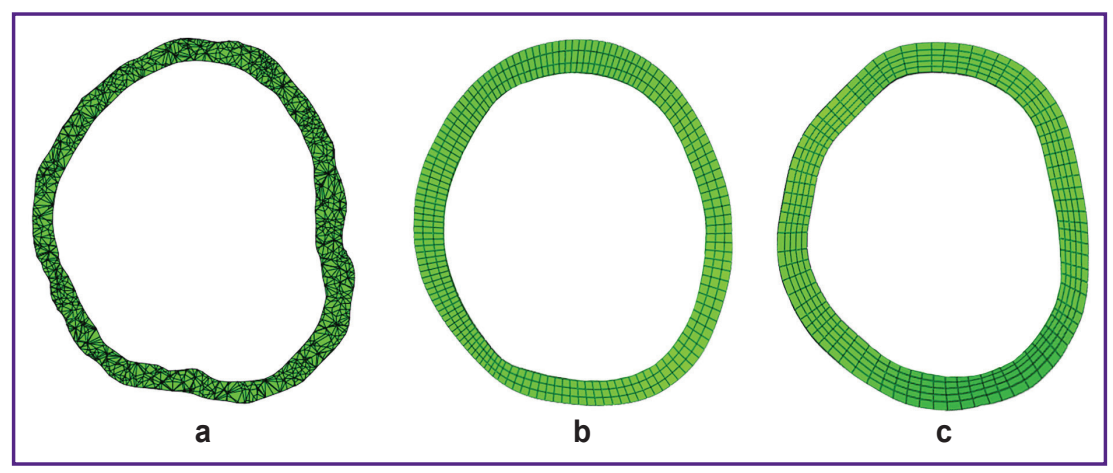

Figure 5. Comparison of slices obtained by three methods under study:

(a) grid resulted from the Amira software; (b) grid created from the CADreconstructed model; (c) finite element mesh created by the proposed method of uniform crushing

accuracy between the models can be exemplified by a slice obtained from the area above the Valsalva sinuses (Figure 5). Here, despite the general similarity between the slice geometry, the CAD method (Figure 5 (b)) produced only rough approximation of the reconstructed area smoothed a number of small details. The proposed numerical method (Figure 5 (c)), although not free from this drawback, better fits the grid constructed by the method of polygons (Figure $5(\mathrm{a})$ ).

The balance between the quality and the cost of this model depends on the requirements and complexity of the numerical experiment, but in cases of multicomponent interaction (e.g. in predicting the TAVI outcomes), both CAD and numerical approaches seem to be rational, since both save time during the most "costly" part of the work - the model reconstruction.

\section{Conclusion}

The qualitative and quantitative results of the study demonstrate the usefulness of the proposed algorithm based on hexahedral finite elements in reconstructing a biological object for the purpose of numerical analysis. Using this algorithm in the TAVI modeling process makes it possible to reduce the time of numerical analysis and increase its accuracy, which may improve the quality of preoperative planning.

Financial support. This study was part of project No.0554-2017-0004 "Development of new technical, tactical and strategic approaches to endovascular diagnosis and treatment of atherosclerosis", implemented in the Research Institute of Cardiovascular Diseases (Kemerovo).

Conflict of interest. There is no conflict of interest.

\section{References}

1. Sardari Nia P., Heuts S., Daemen J., Luyten P., Vainer J., Hoorntje J., Cheriex E., Maessen J. Preoperative planning with three-dimensional reconstruction of patient's anatomy, rapid prototyping and simulation for endoscopic mitral valve repair. Interact Cardiovasc Thorac Surg 2017; 24(2): 163-168, https://doi.org/10.1093/icvts/ivw308.

2. Sindeev S.V., Frolov S.V. Modeling the hemodynamics of the cardiovascular system with cerebral aneurysm. Math
Models Comput Simul 2017; 9(1): 108-119, https://doi. org/10.1134/s2070048217010148.

3. Kilic T., Yilmaz I. Transcatheter aortic valve implantation: a revolution in the therapy of elderly and high-risk patients with severe aortic stenosis. J Geriatr Cardiol 2017; 14(3): 204-217, https://doi.org/10.11909/j.issn.1671-5411.2017.03.002.

4. Bogachev-Prokofiev A.V., Sharifulin R.M., Zubarev D.D., Zhuravleva I.Y., Karaskov A.M. Short term results of transcatheter aortic valve replacement with transaortal approach. Rossijskij kardiologiceskij zurnal 2017; 8: 51-58, https://doi.org/10.15829/1560-4071-2017-8-51-58.

5. Chaturvedi A., Hobbs S.K., Ling F.S., Chaturvedi A., Knight P. MRI evaluation prior to transcatheter aortic valve implantation (TAVI): when to acquire and how to interpret. Insights Imaging 2016; 7(2): 245-254, https://doi.org/10.1007/ s13244-016-0470-0.

6. Bockeria L.A., Alekyan B.G., Pursanov M.G., Mironenko V.A., Bockeria O.L., Makarenko V.N., Zakharchenko A.V., Kosenko A.I., Bazarsadayeva T.S., Sandodze T.S., Alekhina M.A. Transcatheter aortic valve implantation: the first experience in Russia. Grudnaa $i$ serdecno-sosudistaa hirurgia 2011; 2: 4-10.

7. Bokeriya L.A., Gudkova R.G. Serdechnososudistaya khirurgiya - 2015. Bolezni i vrozhdennye anomalii sistemy krovoobrashcheniya [Cardiovascular surgery - 2015. Diseases and congenital anomalies of the circulatory system]. Moscow: NTsSSKh im. A.N. Bakuleva; 2016; 208 p.

8. Zhuravleva I.Y., Bogachev-Prokophiev A.V., Demidov D.P., Karaskov A.M. Transcatheter implantation of mitral valve prostheses: current status of the problem. Kardiologiia 2017; 57(8): 51-59, https://doi.org/10.18087/ cardio.2017.8.10018.

9. Neragi-Miandoab S., Michler R.E. A review of most relevant complications of transcatheter aortic valve implantation. ISRN Cardiol 2013; 2013: 956252, https://doi. org/10.1155/2013/956252.

10. Jung J.I., Koh Y.S., Chang K. 3D printing model before and after transcatheter aortic valve implantation for a better understanding of the anatomy of aortic root. Korean Circ J 2016; 46(4): 588-589, https://doi.org/10.4070/ kcj.2016.46.4.588.

11. Tzamtzis S., Viquerat J., Yap J., Mullen M.J., Burriesci G. Numerical analysis of the radial force produced by the Medtronic-CoreValve and Edwards-SAPIEN after transcatheter aortic valve implantation (TAVI). Med Eng Phys 2013; 35(1): 125-130, https://doi.org/10.1016/j. medengphy.2012.04.009.

12. Wald S., Liberzon A., Avrahami I. A numerical study of the hemodynamic effect of the aortic valve on coronary flow. 
Biomech Model Mechanobiol 2017; 17(2): 319-338, https://doi. org/10.1007/s10237-017-0962-y.

13. Bianchi M., Marom G., Ghosh R.P., Fernandez H.A., Taylor J.R. Jr., Slepian M.J., Bluestein D. Effect of balloonexpandable transcatheter aortic valve replacement positioning: a patient-specific numerical model. Artif Organs 2016; 40(12): E292-E304, https://doi.org/10.1111/aor.12806.

14. Klimenov V.A., Alkhimov Yu.V., Shtein A.M., Kasyanov S.V., Babikov S.A., Batranin A.V., Osipov S.P. The use and development of digital radiography methods for technical non-destructive testing and inspection. Kontrol'. Diagnostika 2013; 13: 31-42.

15. Raut S.S., Liu P., Finol E.A. An approach for patientspecific multi-domain vascular mesh generation featuring spatially varying wall thickness modeling. J Biomech 2015; 48(10): 1972-1981, https://doi.org/10.1016/j. jbiomech.2015.04.006.

16. Pavarino E., Neves L.A., Machado J.M., de Godoy M.F., Shiyou Y., Momente J.C., Zafalon G.F., Pinto A.R., Valêncio C.R. Free tools and strategies for the generation of
3D finite element meshes: modeling of the cardiac structures. Int J Biomed Imaging 2013; 2013: 540571, https://doi. org/10.1155/2013/540571.

17. Yu Z., Wang J., Gao Z., Xu M., Hoshijima M. New software developments for quality mesh generation and optimization from biomedical imaging data. Comput Methods Programs Biomed 2014; 113(1): 226-240, https://doi. org/10.1016/j.cmpb.2013.08.009.

18. Otsu N. A threshold selection method from gray-level histograms. IEEE Trans Syst Man 1979; 9: 62-66, https://doi. org/10.1109/tsmc.1979.4310076.

19. Ovcharenko E.A., Klyshnikov K.U., Glushkova T.V., Burago A.U., Zhuravleva I.U. Nonlinear isotropic material model of human aortic root. Tekhnologii zhivykh sistem 2014; 11(6): 43-47.

20. Krishnamurthy A., Gonzales M.J., Sturgeon G., Segars W.P., McCulloch A.D. Biomechanics simulations using cubic hermite meshes with extraordinary nodes for isogeometric cardiac modeling. Comput Aided Geom Des 2016; 43: 27-38, https://doi.org/10.1016/j.cagd.2016.02.016. 